\section{Forensic odontology, part 1. Dental identification}

\author{
J. Hinchliffe ${ }^{1}$ \\ VERIFIABLE CPD PAPER
}

IN BRIEF
Increases understanding of the role of
the forensic odontologist in the human
identification process.
Draws attention to the interface between
the role of the forensic odontologist and
the practise of dentistry.
Emphasises the importance of accurate,
comprehensive dental records.

This series is based upon fact, experience, and some personal views of the author and gives a brief glimpse of forensic odontological issues with regard to the identification of human remains (to include mass fatality incidents), biting injuries and child abuse. The aim of the first paper is to give the reader greater understanding of the role of the forensic odontologist in the identification of human remains, and emphasise the importance of keeping good quality, accurate and comprehensive dental records. Identification of the deceased greatly assists families and friends at this difficult time, as well as aiding law enforcement agencies; getting it wrong is devastating to families and unacceptable. The dental identification process must be carefully undertaken and relies upon the comparison of information from the antemortem record with findings from the postmortem examination, and the efficiency of this process is dependent on the quality and availability of the dental record. As dental team members it is our responsibility to keep and maintain accurate records of our patients. The resilience of the dental structures to postmortem assault, denture labelling, and teeth as a source of DNA, all contribute to making identification successful. Dental identification is widely used, not only in the single fatality situation, but also in mass fatality incidents and cases of missing persons.

Forensic odontology (forensic dentistry) involves the correct collection, management, interpretation, evaluation and presentation of dental evidence for criminal or civil legal proceedings: a combination of various aspects of the dental, scientific and legal professions.

\section{THE ROLE OF THE \\ FORENSIC ODONTOLOGIST}

The work of the forensic odontologist is varied, but most frequently includes:

- Identification of the deceased (to include the mass disaster situation)

- Age estimation of both the living and deceased

- Bite mark analysis

- Abuse issues

- Fragment comparisons

- Archaeological studies

- Cold cases

- Presenting evidence to the courts.

Forensic Odontologist, New Zealand

Correspondence to: Dr Judy Hinchliffe

Email: judy.hinchliffe@gmail.com

\section{Refereed Paper}

Accepted 15 December 2010

DOI: 10.1038/sj.bdj.2011.146

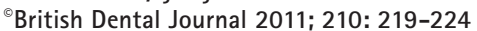

\section{IN GENERAL}

Today, the forensic sciences are closely scrutinised with regard to techniques employed, reliability, validity, accuracy and reproducibility. Forensic odontology, as part of the forensic sciences, must seek to maintain and improve standards and where possible, all work should be based on scientific, accepted, evidencebased procedures and techniques. Also, it is important that the person undertaking the work is a competent (and appropriately trained) forensic odontologist. All opinions and conclusions must be free from personal bias, subjectivity and emotion, and should withstand cross-examination in court. In identification, errors cause confusion and unnecessary distress: the forensic expert carries a weighty responsibility.

Many forensic odontologists worldwide are members of national or international societies and organisations. Some of these organisations are involved with the production of guidance documents and protocols, codes of conduct, mentoring, accreditation, certification, revalidation and peer review. Credit must be given to those associations working hard to move the discipline into the twenty-first century, assisting their members and those utilising the services, and improving credibility and accountability with the public and justice systems. Further research into this subject and education of those working with us (what we can do and how we can assist them) is essential for maintaining standards and moving forward.

\section{INTRODUCTION TO IDENTIFICATION}

The identification of missing or unknown persons is the most common role of the forensic odontologist. Dental identification has proved to be extremely useful and reliable over the years, but is dependent on the availability, adequacy and accuracy of antemortem (AM) dental records that can be compared with the postmortem (PM) dental findings. In the United Kingdom, the forensic odontologist usually enters the investigation at the request of police or coroners (England, Wales and Northern Ireland, procurator fiscal in Scotland), often after other identification methods have been unsuccessful.

Relatives, friends and colleagues of the deceased need proof of identity for many reasons: social, humanitarian, legal, financial, and to enable the body to be disposed 
of in a manner appropriate to that culture. The end of hope may be devastating, but it is believed that a positive identification allows for the grieving process and some degree of closure. When a positive identification has been established, often as a result of multidisciplinary teamwork, investigations (criminal or other) into the circumstances surrounding the death can begin.

It is well known that teeth are of great value for identification purposes because they are one of the most resilient structures in the human body and can survive most conditions of nature that may destroy or change other body tissues. For example, teeth (particularly the posteriors) often survive high temperatures, along with restorations, ${ }^{1-3}$ and sometimes acrylic dentures may survive, ${ }^{4}$ protected by the oro-facial structures. Interventions by the dental team help to make the dentition unique.

\section{Case 1}

A male teenager stole a car after he had been drinking in a local public house. It was dark and he crashed the car on an unlit country lane; the car subsequently caught fire. He was pronounced dead at the scene and his badly burned body removed to the mortuary. Charred clothing and a recovered mobile phone, found in a pocket, were examined and gave a clue to his possible identity. His dental record was requested. At the dental postmortem examination, some of the anterior teeth were found to be fractured and discoloured (Fig. 1) but the posterior teeth were in good condition, revealing several amalgam restorations (Fig. 2). Identification was established after comparing the dental findings with the dental record.

Identification through visual appearance has proved to be unreliable or unacceptable, particularly when there is severe injury, decomposition, skeletalisation (Fig. 3), burning, drowning, mutilation and fragmentation. Personal effects may assist and support identification, but dentistry, fingerprinting, medical information and DNA are the most reliable methods of choice.

\section{Case 2}

Imagine keeping a bedside vigil for your comatose daughter, badly injured in a car accident, only to find that when she starts to recover and drowsily talk, she is not the daughter that you have willed to live, but her friend who looks very similar. Following a crash in America in which five people died (four students and a staff member), the sole survivor was airlifted to hospital after being visually identified by fellow staff and students travelling in another vehicle. The two friends were both blonde with grey-green eyes, of the same build and attended the same university.

As the patient slowly recovered and her injuries continued to heal, she corrected her visiting family, saying her name - not the name the family and boyfriend were expecting. Realisation dawned: this was not their daughter. Their daughter was dead and had already been buried by another family by mistake. Distress for one family, absolute joy for another. This case surely indicates the dangers of relying on visual identification alone. More reliable methods must be used; police, doctors and coroners beware.

\section{Methods used for identification (or to assist with identification)}

\section{Visual (if appropriate)}

2. Personal or medical information:

- General information: height, weight, build, age, presence or absence of hair, its colour and style, eye colour, facial hair, facial characteristics

- Specific information: scars, tattoos, birthmarks, operations, amputations, breast implants, old injuries, medical conditions, body piercings

- Radiological information: anatomical abnormalities, foreign bodies (prostheses)

3. Clothing: items last seen wearing, patterns of fabrics, labels, alterations/ repairs

4. Personal effects and documentation: contents of pockets and bags, jewellery may be recognisable or have specific inscriptions/engravings

5. Dentistry

6. Fingerprints: may be on record, but it is often necessary to take them from personal items in the home or workplace for comparison purposes

7. Feet: footprints are kept on record by some armed forces. Records from a chiropodist/podiatrist may hold useful information

8. DNA profiling.

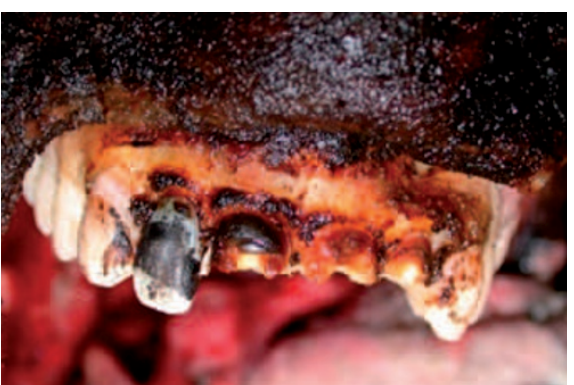

Fig. 1 Anterior teeth fractured and discoloured after car crash and fire

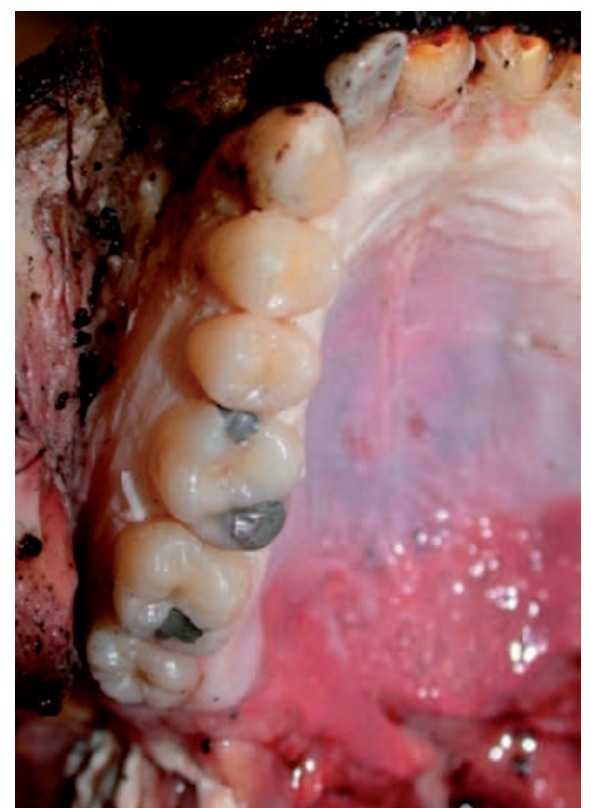

Fig. 2 Same dentition as in Figure 1, showing posterior teeth and restorations protected from fire damage

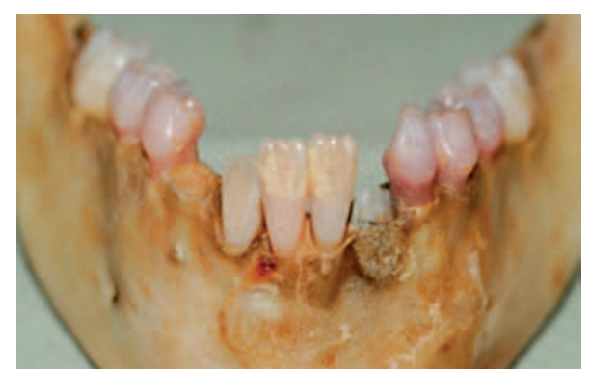

Fig. 3 Skeletalised remains showing the mixed dentition, also narrowing the population search to a child of a certain age range

In many situations, the coroner or investigating officer has a reasonable idea of the identity of the deceased, and uses dental methods for confirmation: comparing the antemortem dental record information with the postmortem dental examination findings (a straightforward comparative identification). When there is no initial clue as to identity, or no antemortem dental record available, the forensic odontologist will make a detailed recording of all 
the dental postmortem findings in case an antemortem record becomes available at a later date. In the absence of any antemortem dental record, the forensic odontologist may work with other forensic disciplines, such as the forensic anthropologist or pathologist, to help narrow the population search by contributing information on the age, sex and ancestry of the deceased, which is sometimes referred to as postmortem dental profiling. ${ }^{5}$

It may be possible to provide information on behavioural habits (for example bruxism), systemic disease, occupation, smoking status and dietary habits. Do the dental features suggest an eating or gastric disorder, alcohol or substance abuse?

\section{THE DENTAL RECORD}

As dentists or dental team members, we know the importance of making and keeping accurate, comprehensive and contemporaneous dental records. Poor records may mean that it is difficult for our defence organisations to defend allegations of clinical negligence or professional misconduct. The antemortem dental record (whether written or computer generated) may provide a wealth of information from:

\section{- Written notes}

- Charts and diagrams

- Medical, dental, social histories

- Radiographs, whether film or digital (and any other images such as MRI))

- Clinical photographs, videos

- Study models

- Referral letters and/or specialist reports

- Results of special tests

- Laboratory prescription: our laboratory colleagues may hold valuable information

- Any other bits of information.

In the United Kingdom, the current recommendation is that dental records should be kept for 11 years, or in the case of a child, until the age of 29 (18 years plus 11). However, Dental Protection would advise that, where possible, records should be kept for life. Claims are often brought against dentists many years after treatment was provided - think implants, periodontal and endodontic treatments particularly!

Good quality dental records are an essential part of patient care, a medicolegal requirement, and are necessary for dental identification. ${ }^{6}$ Their availability and accuracy have a huge impact upon the speed and efficiency with which identification can be accomplished. However, the quality of the antemortem record varies between different dentists, areas and countries.

\section{A few problems encountered with dental records}

- Different charting systems with

different terminology: no international agreement

- Inaccuracies

- Incomplete

- Illegible

- Poor quality of radiographs

- Not transferable in the UK

- Lost or damaged

- Fraudulent.

Resolving the above problems causes delays and confusion, keeping families waiting for news. In the dental undergraduate curriculums (UK), the students base chart all existing treatments as well as those to be carried out to render the patient dentally fit. However, once in the outside world it is not a legal obligation and time constraints mean that the standard sometimes slips (with only the teeth present, absent, and the treatments currently needed to achieve dental fitness being charted), thus losing valuable information. The constantly changing dentition of children, with lack of antemortem radiographs and minimal dental intervention, also cause difficulties with identification.

When dealing with large numbers of fatalities it is practical and sensible to use a standardised, international form to record antemortem and postmortem data so that all participating dentists from around the world use the same terminology and language. The Interpol Disaster Victim Identification (DVI) form is currently widely used, and is being updated at the time of preparing this article with considerable input from forensic dental colleagues around the world. An audit of the standard of the dental records provided by different countries following the Asian Tsunami on Boxing Day 2004 (involving victims from over 35 countries and participating dentists from around the world) would have been most interesting! Article 2 in this series will give an overview of mass fatality incidents.
Good quality radiographs (whether film or digital) are invaluable for revealing 'hidden dental information' along with tooth-coloured restorations that are easily overlooked in less than ideal mortuary conditions. Radiographs may reveal signs of recent or old trauma (accidental or deliberate) that could lead to identification from pins, wires and plates that maxillofacial colleagues may have used. It may be possible to trace the plates and implants (for example) if they have manufacturer's numbers or codes. Postmortem dental radiography can also help with age estimation. It is especially useful for estimating the age at death of children and young adults. However, once the wisdom teeth are fully developed (root formation completed) more invasive methods may be required such as racemisation techniques.

Other useful sources of dental antemortem information are bleaching trays, spare dentures (particularly partial dentures), bite guards, and models retained by the patient, dentist, or dental technician. Dental technicians and medical colleagues may hold valuable information: hospitals may have taken radiographs that include the dental structures. It is important for investigating personnel to check with relatives and other healthcare professionals to ensure the appropriate collection of all useful antemortem information.

\section{Case 3}

The body of a little girl was found in woodland - she had severe head and facial injuries. The police were searching for a particular five-year-old child after she had been reported missing by her worried mother. The child had never been to a dentist, but the mother admitted that her daughter had suffered an untreated abscess on a tooth on the left in the lower jaw. Postmortem dental age estimation confirmed that the child was approximately five years old. Although there were several fractures of the maxilla and mandible, most teeth were present. Clinical examination and postmortem radiography confirmed the presence of a carious lower left deciduous molar with apical pathology. A recent skull radiograph, taken at the local hospital after a fall, also confirmed this carious tooth and along with other information the identification was established. The mother's boyfriend was convicted of manslaughter. 


\section{ANTEMORTEM AND POSTMORTEM COMPARISONS}

When comparing information from the dental antemortem record with the postmortem dental examination findings, there must be a match (no significant differences observed), or any discrepancies should be explainable: perhaps by the passage of time or attendance at a different practice/ hospital for additional treatment (Figs 4a and $4 \mathrm{~b})$. However, there is no minimum number of concordant points: a positive identification can be established from just a few teeth or even a single tooth provided that there are sufficient unique features.

\section{Collection of dental postmortem examination information}

The following will be noted:

- Dental arch shape, alignment, occlusion

- Number and position of teeth present and missing

- Size, shape, position and material of any restorations, presence and position of decayed surfaces

- Denture and other appliance design and material

- Individual tooth characteristics, for example tooth wear, fractures, anomalies of size, shape and colour

- Hard tissue and soft tissue (if present) status, abnormalities or pathologies

- Any other findings of interest, or clues to age, race, diet, occupation etc.

Obviously, it is easier to confirm identity when there has been significant dental intervention (Fig. 5). For example, victims of the Asian Tsunami with numerous and complex dental treatments were quickly identified and returned to their families. When there has been little or no dental intervention it may not preclude identification by dental methods, but may complicate the situation. In the recent Australian bushfires (Victoria), antemortem radiography in one case revealed a healing extraction socket, dip in the alveolar crest and opacities adjacent to the mental foramen - this was all visible in the postmortem radiograph which had been taken from a similar angle. This may not be sufficient for a positive identification, but certainly lends strong support, and along with other information may lead to a positive identification. The training and experience

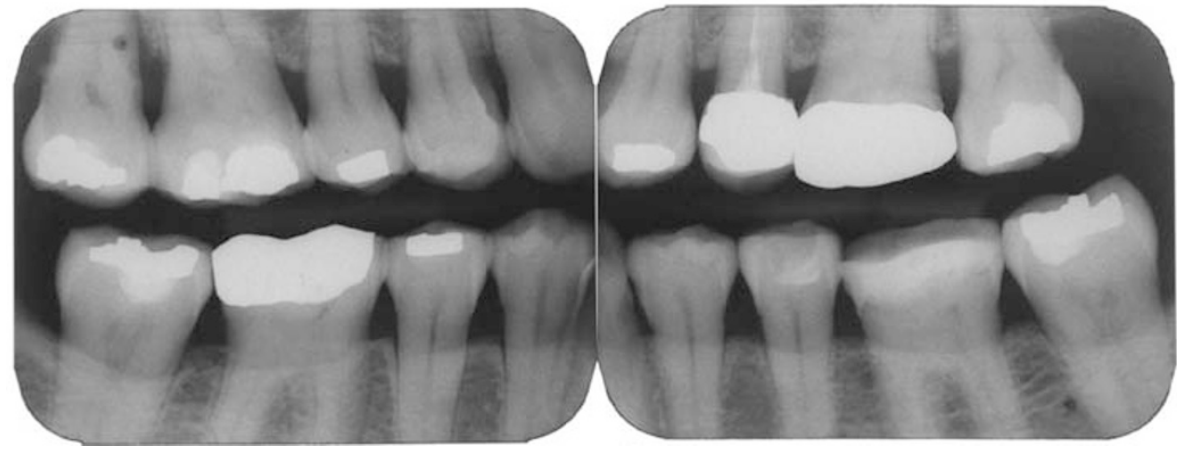

Fig. 4b Postmortem radiographs taken in 2009, showing completed treatment as documented in the dental record: discrepancies explainable and identification established

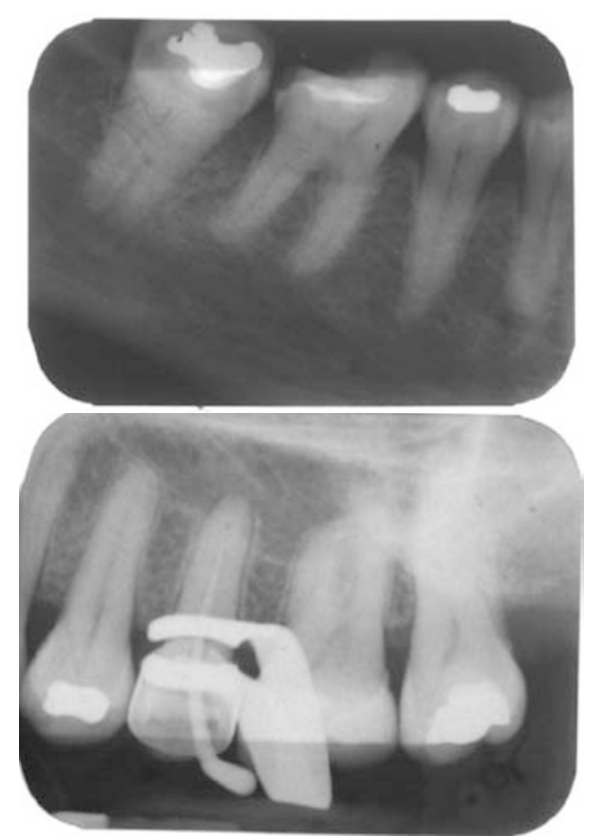

Fig. 4a Following a house fire, the remains of a an adult female were found in a bedroom. Antemortem radiographs taken in 2006

of the odontologist will enable conclusions to be reached that can be justified in court.

\section{POSTMORTEM EXAMINATION DIFFICULTIES}

Mortuary conditions may be less than ideal for dental examinations, so a portable dental forensic kit is essential, and should include a good light source just in case.

Access to the oral cavity may be difficult if rigor mortis is present, the body has been frozen, or is badly burned, when tissues becomes friable and difficult to handle. Gadgets are available to open the jaws without causing damage, or dissection may be necessary, usually following the incisions made by the pathologist and dissecting upwards under the facial tissue. If further examination is needed the jaws may need to be removed (and labelled) and

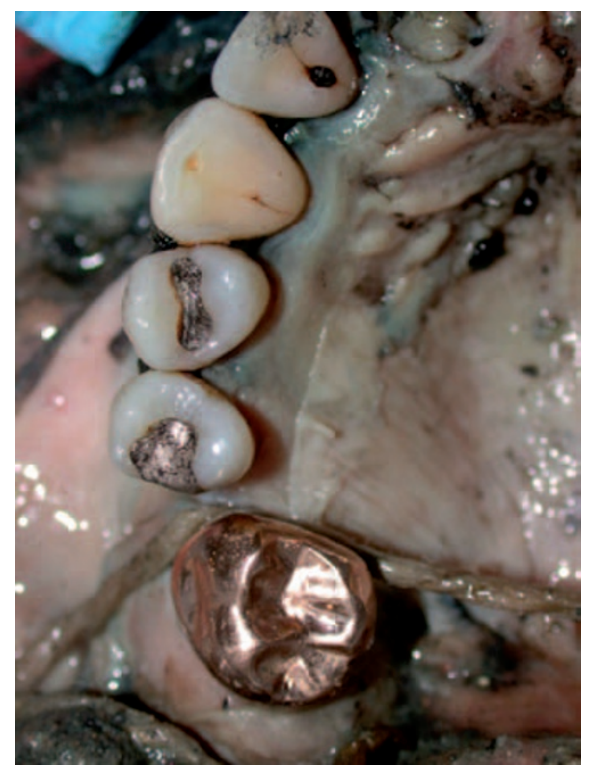

Fig. 5 Restorations still intact, despite extensive damage to other body parts, following a bomb explosion. Courtesy of the Centre for International Forensic Assistance (CIFA)

returned to the body at a later date. When possible (and if necessary), dissection and removal of the mandible intact is most useful and allows for re-articulation. Any and all tissue removed must be discussed with the appropriate authority and only undertaken with relevant consent.

Today, many patients are well-informed about treatment options and aesthetic possibilities. Improving techniques and materials have reduced the use of amalgam in favour of tooth-coloured restorations. It may be extremely difficult to visually distinguish a good quality tooth-coloured restoration from natural tooth structure at the postmortem examination. Various methods have been utilised to detect the presence of these materials, including plaque disclosing solutions, dyes (alizarin red), trans-illumination, and quantitative light-induced fluorescence. ${ }^{7}$ Resins 
fluoresce under certain wavelengths of light: portable ultraviolet (UV) light emitting diode flashlights in the $360 \mathrm{~nm}$ to $385 \mathrm{~nm}$ range can be an adjunctive aid for detecting these restorations. ${ }^{8}$ However, it has been documented that these materials lose their organic components and fluorescence properties after exposure to temperatures above $300^{\circ} \mathrm{C} ;{ }^{9}$ consequently, fluorescence may not reveal restorations in cases of high temperature incineration.

Recent research work has been undertaken $^{10}$ towards compiling a database of the elemental composition of the restorative resins. It has been shown that even after incineration it may still be possible to identify the brand of resin used for the restoration - the inorganic filler particles of the resin are resistant to high temperatures. However, having a database for comparison purposes will only be of use if the brand of material used for the restoration is recorded in the patient's dental record! Different countries may use different materials and an international database may indicate the nationality of the deceased. Similar work and a database is being undertaken on endodontic materials. ${ }^{11}$ However, these specialised methods may be useful in circumstances when the usual, simpler dental identification methodology is not successful.

\section{THE FULL DENTURE}

In some countries denture labelling is mandatory, but unfortunately this is not the case in the UK, so the fully edentulous patient presents a major challenge for dental identification purposes. In an ideal world, all full dentures would have an identifying name or number, not just those belonging to some patients in nursing homes, hospitals and other institutions where mishaps and mix-ups may be expected! However, the denture may demonstrate certain features that may assist with identification, such as old or recent repairs, areas of relief, soft linings, material used, or a particular tooth type and arrangement. If a denture wearer was involved in an accident, crime or mass disaster it would be invaluable if the denture was labelled. ${ }^{12}$ The marker should ideally withstand most conditions, be acceptable to the patient, not weaken the denture and be easy and cheap to produce and give a positive identification.
The results of various experiments with extremely hostile conditions (acid, alkali, liquid nitrogen and crematorium conditions) indicate that stainless steel orthodontic bands included in acrylic dentures survive all the above and are cost-effective, with RFID-tags (radio frequency identification) being a close second. However, with the exception of fire, many basic labelling methods survive a common range of chemical and thermal insults. ${ }^{13}$

\section{HELPFUL DENTAL INFORMATION}

When records are not available or are of poor quality, good quality smiling photographs showing the positions and angles of the anterior teeth may play a part in dental identification. It may be necessary to try facial superimposition techniques or facial reconstruction, and release the results to the public in the hope that information will be forthcoming. Much current research centres around three-dimensional computerised facial reconstruction.

\section{Case 4}

Over a decade ago, I was involved with the case of a 'headless body'. A corpse with no head, no clothes and badly burned, was found by a man walking his dog. At autopsy, the stomach content was sent to the forensic laboratory for analysis and revealed tooth fragments. I was called to examine the fragments which could be identified as parts of an upper anterior porcelain full crown, most likely swallowed by the victim during the incident. Several months later in a different county, a mutilated and decomposed head was discovered in a plastic bag, partially buried in a school playing field. At the scene, a three-unit bridge, with a tooth still attached, was found and collected by crime scene investigators, emphasising the importance of careful and systematic scene searches. The head was matched to our body through DNA analysis. Examination of the head revealed further dental fragments.

Despite publishing the collective dental findings in the dental journals and circulation to local dental practices and hospitals, no identification was made after several months of investigation. I was fortunate to observe the work of an anatomical artist as he reconstructed the features, from skull fragments, in an attempt to further the case. The reconstruction was shown on television (Crimewatch UK) and incoming information from the public gave a potential list of names to work with. One of the names gave us a positive identification through an excellent antemortem dental record, including the presence of porcelain full crowns on three of the upper anterior teeth. Once the identity is established, investigating officers can focus their search for the perpetrator(s) of the crime.

Pieces of tooth (of human origin) or restorations found at crime scenes may link the victim or perpetrator to the scene. To assist with the recognition and differentiation of tooth related fragments, other scientific resources such as microscopy, scanning electron microscopy and energy dispersive X-ray spectroscopy (SEM/EDS) may be necessary.

\section{TEETH AND DNA}

The use of DNA for human identification is well documented ${ }^{14}$ and it has proved to be very effective under many conditions. Teeth can be an excellent source of DNA. ${ }^{15}$ Personal effects can provide a source of DNA as a reference sample for individuals when there is no known or available relative: toothbrushes have been used with success, ${ }^{16}$ also hairbrushes and razors.

The polymerase chain reaction (PCR) technique that enables a minute quantity of DNA to be amplified has had a dramatic impact on the forensic world. Analysis is now possible when there is only a minute amount of DNA recovered from human remains, objects and crime scenes, leading to some 'cold cases' being reopened and solved.

Mitochondrial DNA (maternally inherited, and abundant in bone and teeth) might be useful if nuclear DNA is not available or is too degraded. Factors leading to DNA degradation include time, temperature, humidity, light (both sunlight and UV light), chemical and biological contamination. Low Copy Number (LCN) analysis is an ultra-sensitive technique (sensitive to contamination) useful for trace samples (touched objects, smudges, fingerprints) and those samples that fail standard testing because the sample size is too small or degraded (personal communication from Stephen Cordiner, Institute of Environmental Science and Research Ltd, Porirua, New Zealand). 
Despite the enormous advances with DNA technology, there are situations when it cannot be used and dental methods still contribute hugely to the identification process, currently being relatively quick and economical. It is the responsibility of the forensic odontologist to offer advice and ensure that relevant dental DNA samples have been taken. Scientific colleagues can then analyse and interpret the results.

In 1995, the United Kingdom established the first national DNA database, followed by New Zealand, USA and other countries. The main aim of the database is to assist in the solving of crimes by linking an offender to a crime or crime scene, or linking together various crime scenes. In 2006 the database had helped with more than 700,000 investigations. The use of any database requires a balance of individual civil rights and the interests of the criminal justice system: controversy will always surround the use of such information and protocols need frequent revision. Major issues often relate to criteria for sample entry and retention.

\section{Case 5 - the missing boy}

A young boy was reported missing by his mother in Manchester, UK, in October 1997: he was eight and a half years old. Despite initial searches by police and volunteers, he was not found, but had last been seen playing on a bus. In the summer of the following year, human skeletalised remains were found in woodland close to a fast-flowing river, some miles from the boy's home. No skull was found, just the mandible, parts of the pelvis, some ribs and vertebrae. Clinical and radiographical examination of the mandible gave an age estimation of approximately 8.3 years \pm 3 months. The pathologist confirmed the remains to be those of a young child.

Some posterior deciduous teeth were missing from the mandible, presumed to have been extracted at an earlier date based on the radiographical appearance of the bone. Information from the missing boy's mother suggested that her son was taken to the dentist only when having problems: records were brief with no antemortem radiographs, but did note that the boy had been referred to the local dental school for extractions. The dental school had fortunately retained records from his visit for extractions under a general anaesthetic some years earlier: the posterior deciduous teeth extracted correlated with those missing from the mandible. The dental findings indicated a probable identification of the missing boy.

Genomic DNA testing of the bone and teeth was inconclusive, but mitochondrial DNA testing confirmed that the remains were related to the mother of the missing boy. Suspicion centred on a bus driver (who befriended the family after the disappearance and assisted with searches for the boy) and he was eventually charged with murder. The circumstances of the little boy's death and whereabouts of the rest of his remains are still a mystery.

There is a need for routine odontological input (by dentally qualified personnel) into missing person files and investigations to prevent unnecessary delays in identification. A national missing persons dental records database to assist with the identification process, with links to standardised international databases, would be extremely useful.

\section{CONCLUSION}

The resilience of the teeth and dental structures means that there is much information that can be utilised for the purposes of human identification. For best results, team work is hugely important and we all have a role to play: together, we owe a duty of care and dignity to the deceased, and to assist investigations and the justice systems with thorough and competent examination, evidence collection, interpretation and conclusions. Accurate, comprehensive antemortem dental records are an essential component for successful identification. As dental team members we are in a position to ensure the quality of those records.

\section{Further reading}

Herschaft E E, Alder M E, Ord D K, Rawson R D, Smith E S. Manual of forensic odontology. 4th ed. Lubbock, TX: American Society of Forensic Odontology, 2006

Bowers C M. Forensic dental evidence: an investigator's handbook. USA: Elsevier Academic Press, 2004.

Whittaker D K, MacDonald D G. A colour atlas of forensic dentistry. London: Wolfe Publishing, 1989.

Judy Hinchliffe has been a dental practitioner for many years in South Yorkshire, UK, and had a part-time teaching commitment to the School of Clinical Dentistry, University of Sheffield. She has 17 years of independent forensic casework experience, with involvement in numerous mass fatality incidents. Judy lectures nationally and internationally on forensic dentistry and organises various workshops. She has written articles and contributed to book chapters on forensic dentistry. She is a past president of the British Association for Forensic Odontology, a member of the American Society for Forensic Odontology, the dental council member for the British Association for Human Identification, and was recently made an Honorary Fellow of the new Faculty of Forensic and Legal Medicine, Royal College of Physicians, London. She now divides her time between the UK and New Zealand, and has recently taken over as the editor of the Journal of Forensic Odonto-Stomatology.

1. Carr R F, Barsley R E, Davenport W D. Postmortem examination of incinerated teeth with the scanning electron microscope. J Forensic Sci 1986; 31: 307-311.

2. Merlati G, Danesino P, Savio C, Fassina G, Osculati A, Menghini P. Observations on dental prostheses and restorations subjected to high temperatures: experimental studies to aid identification processes. $J$ Forensic Odontostomatol 2002; 20: 17-24.

3. Merlati G, Savio C, Danesino P, Fassina G, Meghini P. Further study of restored and un-restored teeth subjected to high temperatures. J Forensic Odontostomatol 2004; 22: 34-39.

4. Rotzscher K, Grundmann C, Benthaus S. The effects of high temperatures on human teeth and dentures. Int Poster J Dent Oral Med 2004; 6: Poster 213.

5. Pretty I A, Sweet D. A look at forensic dentistry part 1: the role of teeth in the determination of human identity. Br Dent J 2001; 190: 359-366.

6. Hinchliffe J A. Forensic dentistry: an introduction to identification issues. Dental Tribune Asia Pacific Edition 2007; 11: 8-10.

7. Pretty I A, Smith P W, Edgar W M, Higham S M. The use of quantitative light-induced fluorescence (QLF) to identify composite restorations in forensic examinations. J Forensic Sci 2002; 47: 831-836.

8. Hermanson A S, Bush M A, Miller R G, Bush P J. Ultraviolet light as an adjunctive aid in dental inspection. J Forensic Sci 2008; 53: 408-411.

9. Bush M A, Miller R G, Prutsman-Pfeiffer J, Bush $P$ J. Identification through XRF analysis of dental restorative resin materials: a comprehensive study of non-cremated, cremated, and processed cremated individuals. J Forensic Sci 2007; 52: 157-165.

10. Bush M A, Bush P J, Miller R G. Detection and classification of composite resins in incinerated teeth for forensic purposes. J Forensic Sci 2006; 51: 636-642.

11. Bonavilla J D, Bush M A, Bush P J, Pantera E A. Identification of incinerated root canal filling materials after exposure to high heat incineration. J Forensic Sci 2008; 53: 412-418.

12. Richmond R, Pretty I A. Contemporary methods of labelling dental prostheses - a review of the literature. J Forensic Sci 2006; 51: 1120-1126.

13. Richmond R, Pretty I A. A range of postmortem assault experiments conducted on a variety of denture labels used for the purpose of identification of edentulous individuals. J Forensic Sci 2009; 54: 411-414.

14. Lygo J E, Johnson P E, Holdaway D J et al. The validation of short tandem repeat (STR) loci for use in forensic casework. Int J Legal Med 1994; 107: 77-89.

15. Sweet $D$, Hildebrand D, Phillips D. Identification of a skeleton using DNA from teeth and a PAP smear. J Forensic Sci 1999; 44: 630-633.

16. Tanaka M, Yoshimoto T, Nozawa H et al. Usefulness of a toothbrush as a source of evidential DNA for typing. J Forensic Sci 2000; 45: 674-676. 